\title{
DISTRIBUIÇÃO SAZONAL DE ZOOPLĀNCTON, OVOS E LARVAS DE PEIXES NA REGIÃO CENTRO-SUL DO BRASIL (1975-77)
}

\author{
YASUNOBU MATSUURA, KESHIYU NAKATANI E SERGIO TADEU JUROVSKY TAMASSIA \\ Instituto Oceanográfico da Universidade de São Paulo, São Paulo, SP, Brasil
}

\section{SYNOPSIS}

Abundances of zooplankton volumes, fish eggs and larvae were determined for 812 oceanographic stations on six survey cruises to the southern Brazilian continental shelf. Contour charts of zooplankton volumes are presented. A marked seasonality on zooplankton volumes and number of fish eggs and larvae was observed. The highest values were obtained during late spring and summer seasons and the lowest in the autumn. The mean values of zooplankton volumes, eggs and larvae for different depth zones and subareas were presented. Major part of fish larvae were classified into 55 families. The most abundant group were those of family Engraulidae, Myctophidae and Gonostomatidae. The larvae of Clupeidae were abundant in the late-spring and summer cruises.

\section{Introdução}

$O$ projeto FINEP que é uma investigação integrada de oceanografia, deu uma boa oportunidade de se estudar as distribuiçōes de cooplâncton, ovos e larvas de peixes e sua variação sazonal na região centro-sul do Brasil.

O presente trabalho apresenta os resultados preliminares da análise do zooplâncton, e o comportamento da ocorrência dos ovos e larvas das principais famílias de peixes. Não entramos na análise de cada espécie, mas a variação sazonal de abundância de cada família dará uma noção sobre a abundância relativa de cada grupo.

\section{Material e Método}

O material é proveniente de seis cruzeiros oceanográficos realizados com o N/Oc. "Prof. W. Besnard" do Instituto Oceanográfico da USP, na região entre Cabo Frio $\left(23^{\circ} \mathrm{S}\right)$ e Cabo de Santa Marta Grande $\left(29^{\circ} \mathrm{S}\right)$, (Fig. 1, Tab. I).

As amostras de zooplâncton foram coletadas com rede de plâncton, tipo Bongo (Posgay et al., 1968) e a metodologia de coleta baseou-se na descrição de Ahlstrom et al. (1973). Os materiais coletados foram fixados em solução de formol à $10 \%$, neutralizado. No laboratório, determinou-se o volume de zooplâncton pelo método do deslocamento (Kramer et al., 1972) e a triagem de ovos e larvas de peixes, foi feita com auxílio de lupas estereoscópicas.

Para a distribuição de zooplâncton foram utilizados os dados da rede de malha fina ( 333 micrometros) e no estudo da abundância de ovos e larvas foram usados os da rede de malha regular (505 micrometros).

\section{Resultados}

\section{A) Natureza da distribuiçāo}

Foi calculado a média, erro padrão e o coeficiente de variação para todas as observaçōes (Tab. II). A heterogeneidade na distribuição desses organismos é refletida pelos altos valores do coeficiente de variação e pela distribuição de freqüência da abundância, com todos os dados agrupados (Fig. 2) a aual apresenta alta "Skewness", mostrando uma distribuição assimétrica do tipo L.

\section{B) Sazonalidade da abundância}

Pela Tabela II verificamos que a variação do volume médio de zooplâncton apresentou uma sazonalidade alta. Nas viagens de primavera (Cruz. I e V) e nas viagens de verão (Cruz. II e VI) as médias foram maiores, enquanto que no outono (Cruz. III) foi a menor.

Para o número médio de ovos $/ \mathrm{m}^{2}$, assinalamos a maior ocorrência no Cruzeiro I com 183,83 ovos $/ \mathrm{m}^{2}$, seguida pelo $\mathrm{Cru}$ zeiro IV com 160,04 ovos $/ \mathrm{m}^{2}$ e a menor abundância no Cruzeiro III com 36,67 ovos $/ \mathrm{m}^{2}$.

Em relação ao número médio de larvas $/ \mathrm{m}^{2}$, a variação sazonal foi similar à variação do volume de zooplâncton, com abundância máxima de 97,83 larvas $/ \mathrm{m}^{2}$, no 6 ? Cruzeiro, e mínima de 54,54 larvas $/ \mathrm{m}^{2}$, no 3 ? Cruzeiro.

Esta diferença de sazonalidade entre ovos e larvas é devido a uma distribuição heterogênea dos ovos (patcheness), pois os es- tádios de ovo são relativamente curtos quando comparados com os estádios de larvas, portanto a sua distribuição é mais concentrada nas proximidades da área de desova do que as larvas que sofrem uma maior deriva e se espalham por uma área bem maior.

\section{C) Distribuiçāo por setores}

A área de estudo foi dividida arbitrariamente em três setores, a saber: NORTE (de Cabo Frio até o sul da Iltha de São Sebastião) CENTRO (do sul da llha de São Sebastião até Iltha de São Francisco do Sul) e SUL (da Ilha de São Francisco do Sul até Cabo de Santa Marta Grande), (Fig. 1).

$\mathrm{Q}$ volume médio de zooplâncton $/ \mathrm{m}^{2}$ e o número médio de ovos $/ \mathrm{m}^{2}$ foi maior no setor Centro, com exceção nos cruzeiros de outono e inverno, onde o volume de zooplâncton foi maior no setor Norte e o número de ovos no setor Sul, respectivamente Enquanto no geral o número médio de larvas foi maior no setor Sul, e esta situação se manteve quando analisamos os dados agrupados. E a sazonalidade referenciada anteriormente também está presente em cada um dos setores.

\section{D) Distribuiçāo por isóbatas}

A área de investigação foi dividida em quatro zonas de diferentes profundidades: Zona 1, 20-50 m; Zona II, 51-100 m; Zona III, $101-200 \mathrm{~m}$ e Zona IV com mais de $200 \mathrm{~m}$.

Observando a Tabela IV verificamos que o volume médio de zooplâncton foi bem maior em profundidades menores que $100 \mathrm{~m}$, para a abundância de ovos, em 4 dos 6 cruzeiros, a Zona apresentou maior abundância, sendo as exceçőes nas viagens realizadas no outono e inverno onde as maiores concentrações foram nas Zonas IV e III, respectivamente. As larvas apresentaram' maior abundância na Zona II e a menor na Zona I.

E) Äreas especificas de desova e concentração de zooplâncton

A Figura 3 apresenta a distribuição de zooplâncton observada nos seis cruzieros, mas devido a sua natureza heterogênea, fica difícil determinar-se áreas de maior abundância, mas os dados obtidos sugerem a existência de uma área de alta concentração na região de Santos, durante o verão (Cruz. I-II-V-VI). Analisando a ocorrência de Santos, somos levados a supor que durante o verão a água do mar estando mais quente, determina na região uma grande produção de plâncton, que é constituido principalmente por tunicados e sifonomedusas, mas a grande concentração observada no 1 ? Cruzeiro, em frente a Santos, provavelmente está relacionado às águas interiores da Baixada Santısta (devido às chuvas que ocorreram naquela época). Grandes concentrações ocorreram na altura de Ubatuba, mas sua ocorrência deu-se apenas no fim da primavera (Cruz. I-V)

Somente no Cruzeiro V, na região de Santa Marta Grande, em área costeira, foi constatada uma grande concentração de zooplâncton $\left(1,0 \mathrm{ml} / \mathrm{m}^{3}\right)$, acerca da qual Matsuura (1977), sugeriu a possibilidade de ocorrência de uma ressurgência semelhante à de Cabo Frio, o que explicaria a água fria encontrada nesta época do ano $\left(21^{\circ} \mathrm{C}\right)$.

Para os ovos, devido ao fato de ocorrer desova durante o ano todo, não foi encontrada nenhuma área específica, a qual somente poderia ser determinada com o estudo de cada espécie.

F) Abundância de Larvas

As larvas de peixes foram classificadas até ao nível de família e algumas até o nível de ordem. Das 55 familias identificadas a 
dos Engraulidae, Myctophidae e Gonostomatidae foram as que apresentaram maior abundância durante 0 ano, no entanto as larvas de Clupeidae ocorreram intensivamente durante o veråo (Tab. V).

Os clupeídeos apresentaram uma sazonalidade bem evidente, com desova mais concentrada no fim da primavera e no verão, enquanto que nas outras estaçōes a sua ocorrência é bem reduzida quando comparada comr a dos engraulídeos, que foram constatados durante o ano todo.

Esses resultados,concordam com Matsuura (1977) que constatou ser a época de desova da sardinha-verdadeira, que é a espécie mais abundante da região, durante o verão, chegando seu máximo nos meses de dezembro e janeiro. Os engraulídeos foram as larvas de maior ocorrência nos seis cruzeiros, tendo sido assinaladas em toda a plataforma continental, com uma expressiva distribuição entre as isóbatas de $50-100 \mathrm{~m}$.

As larvas de carangídeos e bregmacerotídeos, preferiram águas mais frias, enquanto que, os scombrídeos foram assinalados, pela primeira vez com expressiva ocorrência. no Cruzeiro VI.

Larvas das familias Myctophidae e Gonostomatidae (peixelanternas), apesar de pertencerem a duas ordens diferentes, foram consideradas como um grupo à parte, a dos peixes mesopelágicos. As larvas deste grupo ocorreram durante o ano todo, especialmente nas regiōes fora da plataforma continental. Sua abundância relativa é sempre equivalente com a dos grupos dos engraulídeos e dos clupeídeos, podendo este grupo ser um importante componente do ictioplâncton do mar aberto.

Tabela I - Relação dos cruzeiros na região centro-sul do Brasil, em 1975-77, e no de observações.

\begin{tabular}{|lcc|}
\hline CRUZEIRO & DATA & OBSERVAÇÕES \\
\hline I & $28 / 11 / 75-19 / 12 / 75$ & 140 \\
II & $08 / 01 / 76-29 / 01 / 76$ & 140 \\
III & $07 / 05 / 76-27 / 05 / 76$ & 140 \\
IV & $22 / 09 / 76-15 / 10 / 76$ & 140 \\
V & $01 / 12 / 76-21 / 12 / 76$ & 140 \\
VI & $17 / 01 / 77-08 / 02 / 77$ & 112 \\
\hline
\end{tabular}

Tabela II - Volume médio de zooplâncton, número médio de larvas e ovos de peixes, para os cruzeiros realizados em 1975-77, na região cen tro-sul do Brasil.

\begin{tabular}{|c|c|c|c|c|}
\hline Cruzeiro & $\mathbf{N}$ & $\overline{\mathbf{x}}$ & $s_{\bar{x}}$ & C.V. \% \\
\hline \multicolumn{5}{|c|}{$\begin{array}{c}\text { ZOOPLĀNCTON } \\
\text { (ml de zooplâncton } / \mathrm{m}^{3} \text { ) }\end{array}$} \\
\hline I & 139 & 0,35 & 0,03 & 100,0 \\
\hline II & 139 & 0,42 & 0,06 & 173,8 \\
\hline III & 140 & 0,15 & 0,01 & 73,3 \\
\hline IV & 140 & 0,24 & 0,01 & 66,7 \\
\hline V & 140 & 0,57 & 0,07 & 152,6 \\
\hline VI & 112 & 0,98 & 0,13 & 136,7 \\
\hline Total & 810 & 1,44 & 0,03 & 170,5 \\
\hline \multicolumn{5}{|c|}{$\begin{array}{l}\text { LARVAS DE PEIXES } \\
\text { (n? de larvas } / \mathrm{m}^{2} \text { de área) }\end{array}$} \\
\hline 1 & 140 & 68,52 & 6,40 & 112,7 \\
\hline II & 139 & 85,90 & 8,94 & 123,2 \\
\hline III & 140 & 54,54 & 4,93 & 106,9 \\
\hline IV & 140 & 68,26 & 6,97 & 120,8 \\
\hline V & 140 & 86,57 & 7,67 & 104,9 \\
\hline VI & 112 & 97,83 & 11,78 & 127,5 \\
\hline Total & 811 & 76,48 & 3,22 & 119,9 \\
\hline \multicolumn{5}{|c|}{$\begin{array}{c}\text { OVOS DE PEIXE } \\
\text { (n! de ovos } / \mathrm{m}^{2} \text { de área) }\end{array}$} \\
\hline I & 140 & 183,87 & 39,90 & 256,8 \\
\hline II & 138 & 121,52 & 20,40 & 198,6 \\
\hline III & 140 & 37,47 & 12,80 & 412,2 \\
\hline IV & 140 & 160,04 & 34,40 & 254,2 \\
\hline V & 140 & 99,99 & 15,90 & 188,1 \\
\hline VI & 112 & 88,28 & 11,50 & 153,6 \\
\hline Total & 810 & 110,94 & 10,25 & 262,0 \\
\hline
\end{tabular}

Tabela III - Valor médio do volume de zooplâncton $\left(\mathrm{ml} / \mathrm{m}^{3}\right.$ de água filtrada) e do número de ovos e larvas de peixe (no $/ \mathrm{m}^{2}$ de superfície do mar) para os cruzeiros de 1975-77, na região centro-sul do Brasil. (Região dividida em três setores).

\begin{tabular}{|c|c|c|c|c|c|c|c|c|c|}
\hline & \multicolumn{3}{|c|}{$\begin{array}{c}\text { ZOOPLĀNCTON } \\
\left(\mathrm{ml} / \mathrm{m}^{3}\right)\end{array}$} & \multicolumn{3}{|c|}{$\begin{array}{l}\text { LARVAS } \\
\left(\text { larvas } / \mathrm{m}^{2} \text { ) }\right.\end{array}$} & \multicolumn{3}{|c|}{$\begin{array}{c}\text { ovos } \\
\left(\text { ovos } / \mathrm{m}^{2}\right)\end{array}$} \\
\hline & NORTE & CENTRO & SUL & NORTE & CENTRO & SUL & NORTE & CENTRO & SUL \\
\hline 1 & 0,26 & 0,52 & 0,27 & 61,94 & 60,73 & 84,49 & 170,47 & 262,50 & 110,92 \\
\hline II & 0,26 & 0,78 & 0,26 & 84,32 & 105,07 & 69,84 & 137,53 & 96,48 & 93,10 \\
\hline III & 0,16 & 0,15 & 0,13 & 57,24 & 45,43 & 60,60 & 41,15 & 12,58 & 14,19 \\
\hline IV & 0,27 & 0,22 & 0,23 & 41,56 & 59,81 & 108,24 & 64,67 & 134,42 & 257,18 \\
\hline $\mathrm{v}$ & 0,53 & 0,67 & 0,53 & 88,39 & 106,57 & 65.73 & 94,17 & 126,46 & 80,01 \\
\hline VI & 0,86 & 1,32 & 0,46 & 81,68 & 106,76 & 128,80 & 84,83 & 97,07 & 62,90 \\
\hline Médio & 0,39 & 0,61 & 0,31 & 69,19 & 80,73 & 86,28 & 98,80 & 121,59 & 107,63 \\
\hline
\end{tabular}


Tabela IV - Sumário dos dados de volume médio de zooplâncton, número médio de ovos e de largas de peixes, após a divisão da área de estudo em quatro zonas, de acordo com a profundidade, para os cruzeiros na região centro-sul do Brasil, em $1975-77$.

\begin{tabular}{|c|c|c|c|c|c|c|c|c|c|c|c|c|}
\hline & \multicolumn{4}{|c|}{$\begin{array}{l}\text { ZOOPLÃNCTON } \\
\left(\mathrm{ml} / \mathrm{m}^{3}\right)\end{array}$} & \multicolumn{4}{|c|}{$\begin{array}{c}\text { LARVAS } \\
\left(\mathrm{n} \text { ? de larvas } / \mathrm{m}^{2}\right)\end{array}$} & \multicolumn{4}{|c|}{$\begin{array}{c}\text { Ovos } \\
\left(\mathrm{n} \text {. de ovos } / \mathrm{m}^{2}\right)\end{array}$} \\
\hline & ZONA I & ZONA II & ZONA III & ZONA IV & ZONA I & ZONA II & ZONA III & ZONA IV & ZONA 1 & ZONA II & ZONA III & ZONA IV \\
\hline I & 0,45 & $Q, 46$ & 0,30 & 0,07 & 33,70 & 94,11 & 65,64 & 89,40 & 358,86 & 191,41 & 82,65 & 75,90 \\
\hline II & 0,34 & 0,74 & 0,40 & 0,09 & 36,37 & 115,45 & 90,73 & 103,79 & 169,87 & 142,70 & 45,37 & 93,87 \\
\hline III & 0,19 & 0,18 & 0,14 & 0,06 & 14,48 & 43,18 & 100,67 & 48,64 & 19,92 & 10,31 & 23,01 & 55,28 \\
\hline IV & 0,25 & 0,32 & 0,23 & 0,13 & 16,23 & 86,64 & 91,18 & 79,12 & 151,68 & 86,04 & 263,54 & 32,63 \\
\hline V & 0,85 & 0,77 & 0,39 & 0,12 & 71,22 & 123,70 & 64,95 & 96,65 & 166,63 & 103,58 & 74,34 & 38,91 \\
\hline VI & 0,82 & 1,97 & 0,50 & 0,20 & 95,83 & 83,43 & 118,55 & 85,09 & 129,51 & 81,79 & 76,34 & 41,60 \\
\hline Médio & 0,48 & 0,74 & 0,34 & 0.11 & 44,62 & 91,09 & 88,62 & 83,78 & 166,08 & 102,97 & 94,21 & 56,35 \\
\hline
\end{tabular}

Tabela V - Número de larvas capturadas, em cada um đos cruzeiros, na regiảo centro-sul do Brasil em 1975-77).

\begin{tabular}{|c|c|c|c|c|c|c|c|c|c|}
\hline 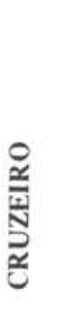 & 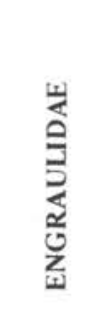 & $\frac{\substack{1 \\
\frac{1}{2}}}{5}$ & 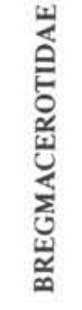 & 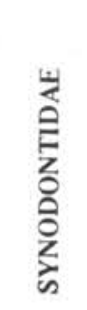 & $\frac{\sqrt{1}}{0}$ & 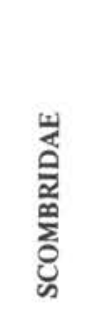 & 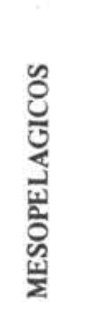 & 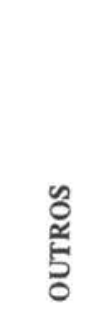 & $\underset{6}{\stackrel{2}{6}}$ \\
\hline 1 & 9180 & 7061 & 404 & 1043 & 1105 & 188 & 6787 & 5942 & 31696 \\
\hline II & 6647 & 11830 & 394 & 1438 & 688 & 1139 & 8215 & 7396 & 37747 \\
\hline III & 3662 & 722 & 5885 & 306 & 156 & 2 & 4379 & 4906 & 20013 \\
\hline IV & 10291 & 235 & 1232 & 880 & 2484 & 489 & 4449 & 9806 & 29866 \\
\hline V & 4290 & 9685 & 1387 & 1431 & 2196 & 197 & 6183 & 11522 & 36891 \\
\hline VI & 5129 & 8259 & 156 & 536 & 553 & 1940 & 3877 & 6038 & 26488 \\
\hline
\end{tabular}

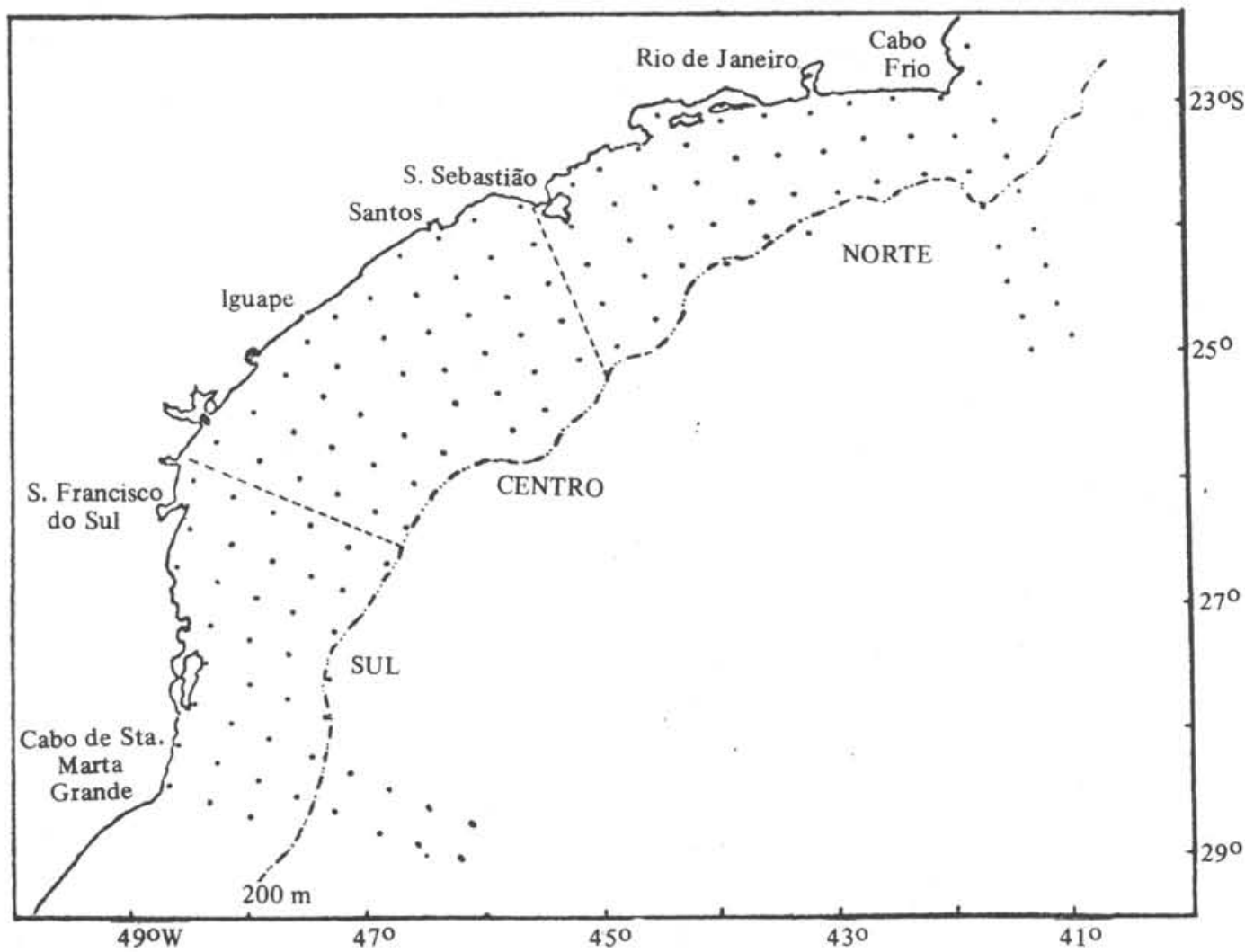

Fig. 1 - Plano das estações e divisão da área em estudo, em três setores. 


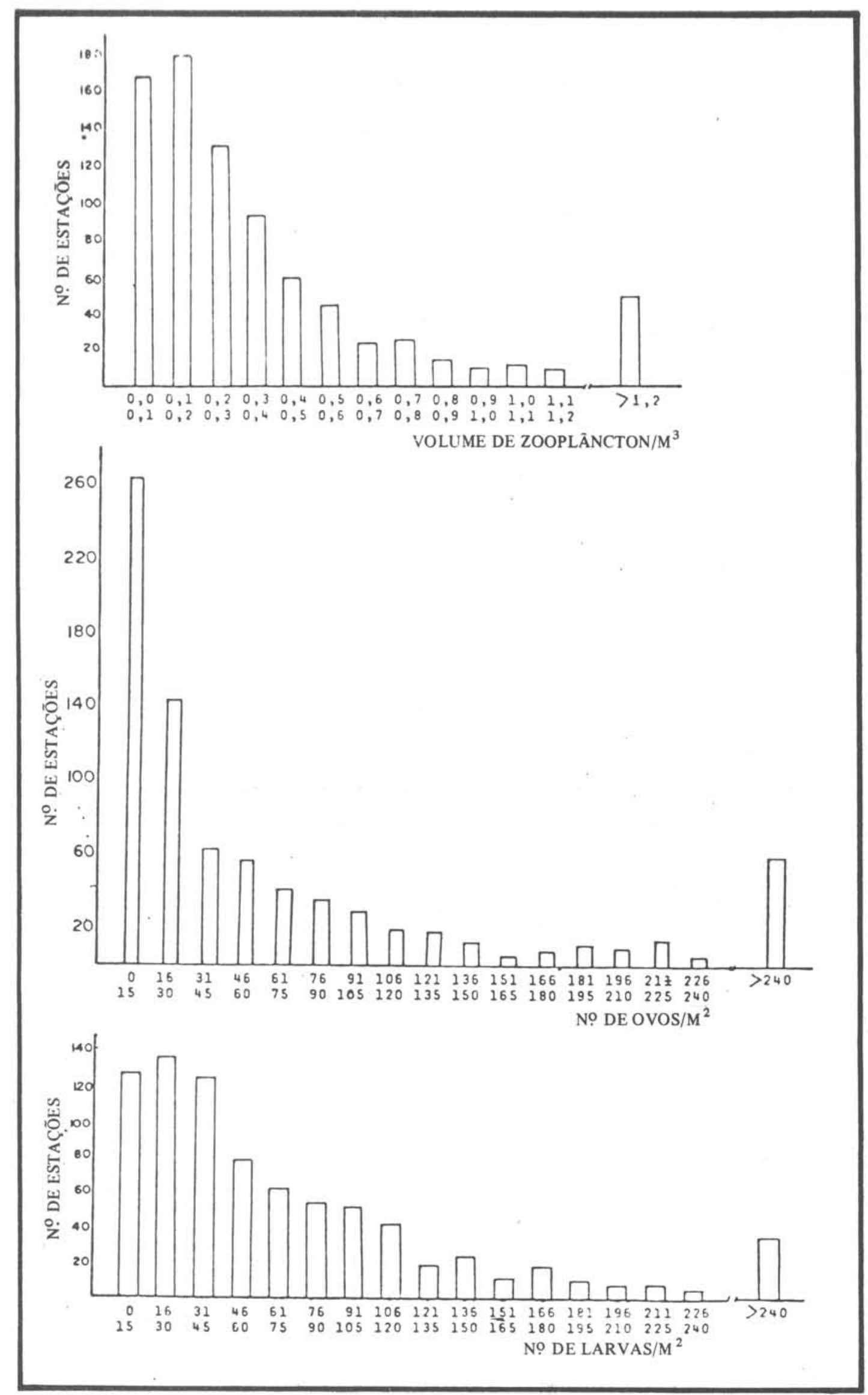

Fig.2 - Distribuição de freqüência do no médio do volume de zooplâncton, $n$ ? de ovos e larvas de peixes, com dados de todos os cruzeiros agrupados, para a região centro-sul do Brasil, em 1975-77. 

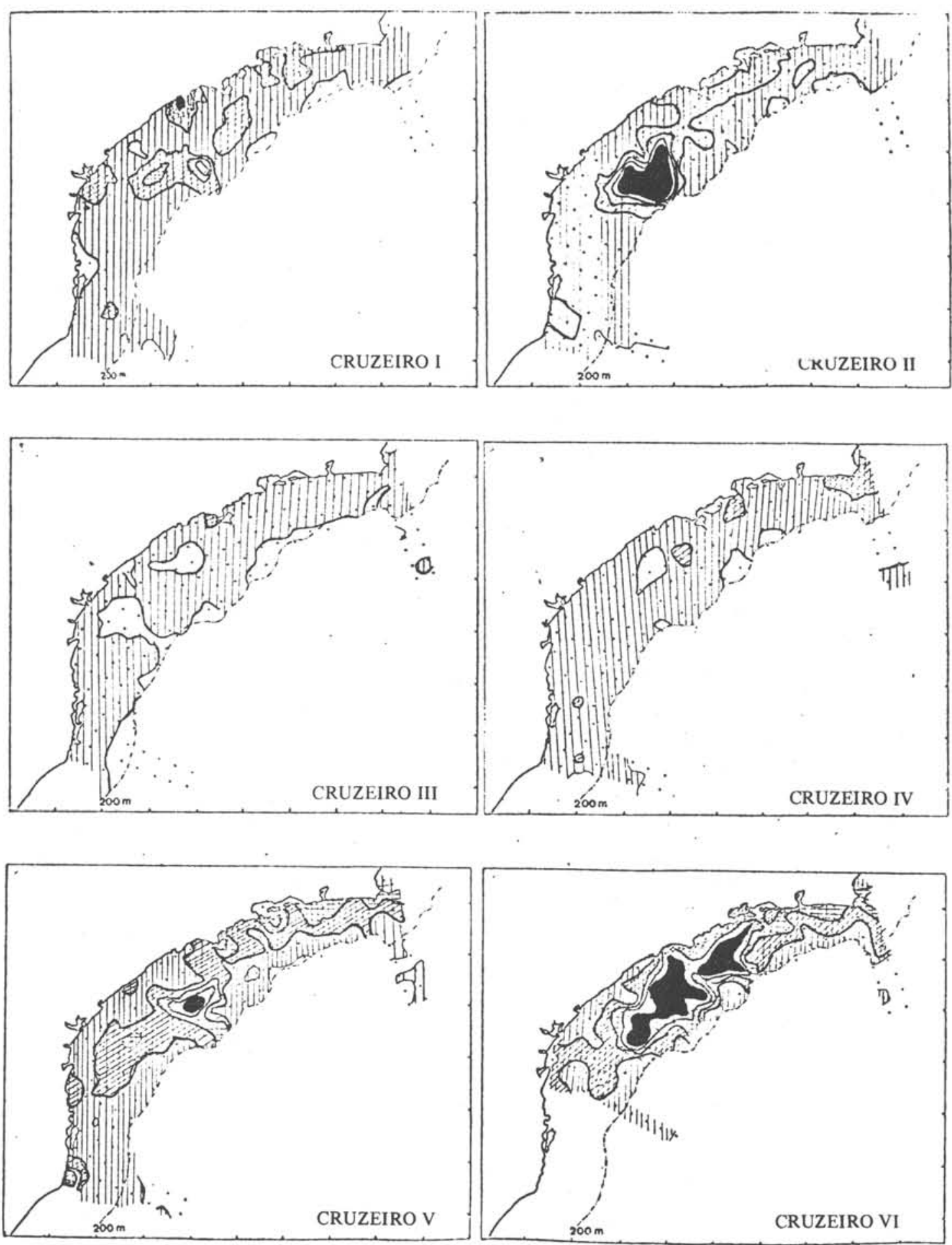

Fig. 3 - Distribuição da abundância do zooplâncton, observada nas viagens de 1975-77. (Malha $333 \mu$ ),

$$
\text { (1) } \begin{array}{r}
<0,1 \mathrm{ml} / \mathrm{m}^{3} \\
0,1-0,5 \mathrm{ml} / \mathrm{m}^{3} \\
0,5-1,0 \mathrm{ml} / \mathrm{m}^{3}
\end{array}
$$

$1,0-1,5 \mathrm{ml} / \mathrm{m}^{3}$

$1,5-2,0 \mathrm{ml} / \mathrm{m}^{3}$
$>2,0 \mathrm{ml} / \mathrm{m}^{3}$

\section{Bibliografia}

AHLSTROM, E. H.; SHERMAN, K. \& SMITH, P. E. 1973. Seagoing operations in ichthyoplankton surveys. F.A.O. Fish. tech. Pap., (122): 14-26.

KRAMER, D.; KALIN, M. J.; STEVENS, E. G.; THRAILKILL, J. R. \& ZWEIFEL, J. R. 1972. Collecting and processing data on fish eggs and larval in the California Current region. NOAA Tech. Resp., NMFS Circ., (370): 1-38.

MATSUURA, Y. 1977. O ciclo de vida da sardinha-verdadeira (Introdução à oceanografia pesqueira). Publ. esp. Inst. Oceanogr. S. Paulo, (4): 1-146.

POSGAY, J. A.; MARAK, R. R. \& HENNEMUTH, R. C. 1968. Development and test of new zooplankton samplers. A Metg int. Commn NW. Atlant. Fish., Res. Doc., (85) (mimeo). 